\title{
THE ELECTROMAGNETIC SECTOR OF THE EVANS FIELD THEORY
}

\author{
M. W. Evans \\ Alpha Institute for Advanced Study (AIAS) \\ E-mail:emyrone@aol.com
}

Received 7 April 2004; revised 8 May 2004

The equations of the electromagnetic sector of the Evans field theory are given in terms of differential geometry and are based on the wellknown structure relations and Bianchi identities. The equations thus complete Einstein's basic axiom, that physics is derived from geometry, and extend the axiom to electrodynamics. Precise tests are suggested for the theory using the interaction of circularly polarized electromagnetic radiation with a non-relativistic electron beam. These tests include; the inverse Faraday effect (IFE), radiatively induced fermion resonance (RFR), and the electromagnetic Aharonov-Bohm (EMAB) effect.

Key words: the Evans field theory, electromagnetic sector, tests of the Evans theory, inverse Faraday effect, radiatively induced fermion resonance, electromagnetic Aharonov-Bohm effect.

\section{INTRODUCTION}

Recently the first successful generally covariant unified field theory has been developed and can be applied to all radiated and matter fields [120]. In Sec. 2 of this paper we give the equations of the electromagnetic sector of the theory and show that they are closely based on well known equations of differential geometry: the structure relations and Bianchi identities [21]. In Sec. 3, high precision tests of the theory are proposed in a non-relativistic electron beam interacting with circularly polarised electromagnetic radiation: the inverse Faraday effect (IFE), radiatively induced fermion resonance (RFR), and the electromagnetically induced 
Aharonov Bohm (EMAB) effect.

\section{EQUATIONS OF THE ELECTROMAGNETIC SECTOR OF EVANS' FIELD THEORY}

In this section we will give the equations of the electromagnetic sector of the Evans theory [1-20] in a form which is easily recognisable as standard differential geometry. The latter is transformed into Evans electromagnetic field theory using the ansatz [1-20]

$$
A_{\mu}^{a}=A^{(0)} q_{\mu}^{a}
$$

Equation (1) defines the electromagnetic potential field $A_{\mu}^{a}$ as a vectorvalued tetrad one-form within a $C$ negative factor $A^{(0)}$. The tetrad $q_{\mu}^{a}$ [1-21] is the gravitational potential field, so the ansatz (1) illustrates field unification in the simplest way possible. In order to introduce the notation, we first give the older Maxwell-Heaviside (MH) field equations of special relativistic electrodynamics. In this way the advantages of, and the new information contained in, the new theory can be seen the most clearly.

The MH equations are expressed most eloquently in terms of differential forms $[21,22]$ as follows:

$$
\begin{gathered}
F=d \wedge A, \\
d \wedge F=0, \\
d \wedge \widetilde{F}=\mu_{0} J .
\end{gathered}
$$

Here $A$ is the scalar-valued potential one-form, $d \wedge$ the exterior derivative of differential geometry, $F$ the scalar-valued two-form that encapsulates the gauge-invariant electromagnetic field, $\widetilde{F}$ the dual of $F$ $[21,22]$, and $J$ the scalar-valued three-form that encapsulates the charge current density of $\mathrm{MH}$ field theory. Here $\mu_{0}$ is the permeability in vacuum, and SI units [1-20] have been used.

Equation (3) is a Jacobi identity [22], the most concise form of the homogeneous field equation $\mathrm{MH}$ field theory. The homogeneous field equation summarizes Gauss's law and Faraday's law of induction. These two laws are therefore geometrical identities within a $C$ negative factor that turns the geometry into field equations of physics. This inference gives us a clue as to the geometrical origin of electrodynamics and leads us eventually to the Evans field equations given later in this section. Equation (4) is the inhomogeneous field equation of $\mathrm{MH}$ field theory but in this theory cannot be clearly recognised as an identity of geometry. The inhomogeneous field equation contains Coulomb's and 
the Ampère-Maxwell law. The gauge invariance of MH field theory follows from the Poincaré lemma [22]

$$
d \wedge d=0 .
$$

The electromagnetic two-form is gauge invariant because

$$
d \wedge(d \wedge A)=0
$$

and so $F$ is known as the gauge invariant electromagnetic two-form. Therefore, in the concise and elegant notation of differential geometry, gauge invariance is simple to comprehend, it is the consequence of another geometrical identity, the Poincaré lemma. This suggests that the inhomogeneous field equation should also be a geometrical identity, and we will show later in this section that this is indeed the case in the Evans field theory of general relativity, but not in the $\mathrm{MH}$ field theory of special relativity. In the latter theory the inhomogeneous field equation is empirical, i.e., based on data and not inferred from geometry, as required by the Einsteinian principles of general relativity [23].

For clarity of exposition and as an introduction to the Evans field equations of electrodynamics, we give the well-known [1-22] tensor and vector forms Eqs. (2) to (4) as follows:

The tensor form of Eq. (2) is

$$
F_{\mu \nu}=-F_{\nu \mu}=\partial_{\mu} A_{\nu}-\partial_{\nu} A_{\mu}
$$

and defines the anti-symmetric field tensor $F_{\mu \nu}$ in terms of the potential field $A_{\mu}$, a covariant four-vector. Equation (7) can also be written in contravariant form:

$$
F^{\mu \nu}=-F^{\nu \mu}=\partial^{\mu} A^{\nu}-\partial^{\nu} A^{\mu}
$$

The Jacobi identity (3) in tensor notation is

$$
\partial^{\lambda} F^{\mu \nu}+\partial^{\mu} F^{\nu \lambda}+\partial^{\nu} F^{\lambda \nu}=0 .
$$

On defining the dual tensor

$$
\widetilde{F}^{\mu \nu}=\frac{1}{2} \epsilon^{\mu \nu \rho \sigma} F_{\rho \sigma},
$$

the Jacobi identity (9) becomes the well-known homogeneous field equation of $\mathrm{MH}$ field theory:

$$
\partial_{\mu} \widetilde{F}^{\mu \nu}=0 .
$$


Equation (11) is Eq. (3) in tensor notation.

In defining the dual tensor the totally antisymmetric unit tensor In four dimensions, $\epsilon^{\mu \nu \rho \sigma}$, has been used as usual. This tensor is the same for any non-Euclidean spacetime [21], but in the MH field theory is used in flat or Minkowski spacetime [1-23].

The inhomogeneous field equation in tensor notation is, in standard SI units,

$$
\partial_{\mu} F^{\mu \nu}=\mu_{0} j^{\nu}
$$

where $j^{\nu}$ is the charge current density four-vector. Equation (12) is the tensor equivalent of the form equation (4).

The homogeneous field equation (11) is equivalent to the following two vector equations:

$$
\begin{gathered}
\nabla \cdot \mathbf{B}=0, \\
\nabla \times \mathbf{E}+\frac{\partial \mathbf{B}}{\partial t}=\mathbf{0},
\end{gathered}
$$

respectively Gauss's law and Faraday's law of induction, where $\mathbf{B}^{(3)}$ is magnetic flux density and $\mathbf{E}$ is electric field strength. Here $c$ is the speed of light in vacuum. The inhomogeneous field equation (12) is equivalent to the following two vector equations:

$$
\begin{gathered}
\nabla \cdot \mathbf{D}=\rho, \\
\nabla \times \mathbf{H}=\mathbf{J}+\frac{\partial \mathbf{D}}{\partial t},
\end{gathered}
$$

respectively Coulomb's law and the Ampère-Maxwell law. Here $\mathbf{H}$ is the magnetic field strength, $\mathbf{D}$ the electric displacement, $\rho$ the charge density, and $\mathbf{J}$ the current density.

The great MH field theory, which these well-known equations describe, has held sway in electrodynamics for over a hundred years, but is not a theory of general relativity. The theory is the archetypical theory of special relativity, in which spacetime is Minkowski spacetime, sometimes referred to as flat spacetime. It is well-known that special relativity evolved in 1905 from these same equations.

The Evans field theory infers electrodynamics from the twisting of spacetime and makes electrodynamics a theory of general relativity. Therefore, the Evans theory is the direct logical outcome of the Einsteinian principle that all theories of physics are theories of general relativity, i.e., all of physics is based on geometry. The most eloquent expression of geometry is differential geometry [1,22], which is valid for all spacetimes, and is more general [21] than the Riemannian geometry available to Einstein, a geometry in which the torsion tensor is assumed to be zero when constructing the relation between the Christoffel symbol and the symmetric metric tensor. In particular, differential geometry allows for the existence of the torsion tensor [21] as well as the 
Riemann or curvature tensor, and this fact has been utilized in the development of Evans field theory [1-20] to show that gravitation is the curving of spacetime, electromagnetism is the twisting of spacetime. The torsion tensor becomes the vector-valued torsion two-form $R_{b \mu \nu}^{a}$ in differential geometry. One of the great advantages of differential geometry is that it is valid for all possible spacetimes, so we can drop the indices $\mu \nu$ and simplify the notation [21], thus emphasising the most important principles of the geometry and thence the physics. In other words, we find all the laws of physics from the laws of geometry and take Einstein seriously. The torsion form becomes $T^{a}$ and the Riemann form becomes $R_{b}^{a}$. The second major advantage is that differential geometry is developed in terms of the vector-valued tetrad one form $q_{\mu}^{a}$ which is more fundamental than the metric tensor $g_{\mu \nu}$ used by Einstein because

$$
g_{\mu \nu}=q_{\mu}^{a} q_{\nu}^{b} \eta_{a b}
$$

In other words, the metric tensor is defined as the dot product of two tetrads, so the tetrad factorizes the metric tensor of the base manifold (non-Euclidean spacetime). Here $\eta_{a b}$ is the diagonal metric tensor

$$
\eta_{a b}=\eta^{a b}=\left(\begin{array}{cccc}
1 & 0 & 0 & 0 \\
0 & -1 & 0 & 0 \\
0 & 0 & -1 & 0 \\
0 & 0 & 0 & -1
\end{array}\right)
$$

of the orthonormal spacetime of the tetrad, a flat or Minkowski spacetime. We may therefore express all the important equations in the flat, orthonormal, spacetime, remembering that each equation applies for the unwritten subscript $\mu \nu$ [21] of the non-Euclidean base manifold. In this way we find the equations of generally covariant unified field theory [1-20] by following the differential geometry. Similarly, Einstein discovered the equations of gravitation by following Riemannian geometry.

The field equations of electromagnetism in the Evans theory are found from the following fundamental and well-known equations of differential geometry. The first Maurer-Cartan structure relation [121] defines the vector-valued torsion two-form as the covariant exterior derivative (denoted by $D \wedge$ ) of the tetrad

$$
T^{a}=D \wedge q^{a}=d \wedge q^{a}+\omega_{b}^{a} \wedge q^{b},
$$

where $\omega_{b}^{a}$ is the spin connection [21]. Using the ansatz (1) and multi- 
plying both sides of Eq. (19) by $A^{(0)}$, we obtain

$$
\begin{aligned}
F^{a} & =D \wedge A^{a} \\
& =A^{(0)} T^{a} .
\end{aligned}
$$

This is the equation defining the gauge-invariant electromagnetic field $F^{a}$ in terms of the potential in the Evans theory and is the generally covariant form of Eq. (2) of the $\mathrm{MH}$ field theory of special relativity. By comparison of Eqs. (20) and (2), it is seen that $d \wedge$ has been replaced by $D \wedge, A$ by $A^{a}$, and $F$ by $F^{a}$. The potential and the field have each developed an internal index of the type normally associated with Yang-Mills gauge field theory [22]. We are now able to see, furthermore, that index is the direct result of differential geometry. Thus $A^{a}$ is the potential field of the electromagnetic sector of Evans field theory, and $F^{a}$ is the gauge-invariant field. In tensor notation, $A_{\mu}$ becomes $A_{\mu}^{a}$, a covariant four-vector with an internal index, and $F_{\mu \nu}$ becomes $F_{\mu \nu}^{a}$, an antisymmetric tensor with an internal index. In Eq. (20), the SI units of $F^{a}, A^{a}$, and $D \wedge$ are tesla $=\mathrm{JsC}^{-1} \mathrm{~m}^{-2}$, tesla $m$, and $\mathrm{m}^{-1}$, respectively. If $a=(1),(2),(3)$ of the complex circular basis, then we obtain the field equations of $\mathrm{O}(3)$ electrodynamics [1-20]. The latter have been extensively developed theoretically and tested experimentally. In Sec. 3, we summarize three further tests so we have derived $\mathrm{O}(3)$ electrodynamics from differential geometry using the ansatz (1). In so doing, we have tested the ansatz experimentally, because $\mathrm{O}(3)$ electrodynamics has been tested experimentally [1-20].

The homogeneous and inhomogeneous field equations of the Evans theory are found from the first and second Bianchi identities of differential geometry [21]:

$$
\begin{aligned}
& D \wedge R_{b}^{a}=0, \\
& D \wedge T^{a}=R_{b}^{a} \wedge q^{b} .
\end{aligned}
$$

In terms of the spin connection, Eq. (22) becomes

$$
d \wedge R_{b}^{a}+\omega_{c}^{a} \wedge R_{b}^{c}-R_{c}^{a} \wedge \omega_{b}^{c}=0
$$

and is a generalization of the well-known Bianchi identity of Riemannian geometry:

$$
D_{\rho} R_{\lambda \mu \nu}^{\kappa}+D_{\mu} R_{\lambda \nu \rho}^{\kappa}+D_{\nu} R_{\lambda \rho \mu}^{\kappa}=0 .
$$

In terms of the spin connection, Eq. (23) reads

$$
d \wedge T^{a}+\omega_{b}^{a} \wedge T^{b}=R_{b}^{a} \wedge q^{b}
$$

and is a generalization of the following identity in Riemannian geometry:

$$
R_{\rho \sigma \mu \nu}+R_{\rho \mu \nu \sigma}+R_{\rho \nu \sigma \mu}=0 .
$$


Equation (27) is equivalent to the following fundamental symmetries of the Riemann tensor:

$$
\begin{aligned}
& R_{\rho \sigma \mu \nu}=-R_{\sigma \rho \mu \nu}, \\
& R_{\rho \sigma \mu \nu}=-R_{\rho \sigma \nu \mu} .
\end{aligned}
$$

The structure of the electromagnetic sector of the Evans theory is therefore summarized as follows:

$$
\begin{array}{r}
F=d \wedge A, \\
d \wedge F=0, \\
d \wedge \widetilde{F}=\mu_{0} J .
\end{array} \longrightarrow \quad \begin{array}{r}
T^{a}=D \wedge q^{a}, \\
D \wedge T^{a}=R_{b}^{a} \wedge q^{b} \\
D \wedge R_{b}^{a}=0 .
\end{array}
$$

The homogeneous field equation of the electromagnetic sector of the Evans unified field theory is

$$
D \wedge F^{a}=R_{b}^{a} \wedge A^{b}=A^{(0)} R_{b}^{a} \wedge q^{b}=\mu_{0} j^{a} .
$$

When the electromagnetic and gravitational fields decouple:

$$
\begin{aligned}
& D \wedge F^{a}=0, \\
& R_{b}^{a} \wedge q^{b}=0 ;
\end{aligned}
$$

and, in the $\mathrm{MH}$ limit,

$$
D \wedge F^{a} \rightarrow d \wedge F=0 .
$$

When the gravitational field influences the electromagnetic field or vice versa, the unified field is governed by the equation

$$
D \wedge F^{a}=A^{(0)} R_{b}^{a} \wedge q^{b} \neq 0,
$$

and the homogeneous field equation of the MH theory, Eq. (33), becomes the homogeneous field equation of the Evans unified field theory, Eq. (34).

Equation (31) is the intermediate step towards the completed unified field theory; and, when $a(1),(2),(3)$, Eq. (31) becomes the homogeneous field equation of $\mathrm{O}(3)$ electrodynamics [17]. In vector notation, Eq. (31) becomes the following six equations:

$$
\begin{aligned}
\nabla \cdot \mathbf{B}^{(1)} & =0, \\
\nabla \cdot \mathbf{B}^{(2)} & =0, \\
\nabla \cdot \mathbf{B}^{(3)} & =0, \\
\nabla \cdot \mathbf{E}^{(1)}+\partial \mathbf{B}^{(1)} / \partial t & =0, \\
\nabla \cdot \mathbf{E}^{(2)}+\partial \mathbf{B}^{(2)} / \partial t & =0, \\
\partial \mathbf{B}^{(3)} / \partial t & =0 .
\end{aligned}
$$


In order to search experimentally for the influence of the gravitational field on the electromagnetic field, it is necessary to search for departures from Gauss's law and Faraday's law of induction. Equation (34) is obtained from Eq. (33) by symmetry building ("inverse symmetry breaking"), as summarized in the following diagram: Similarly the

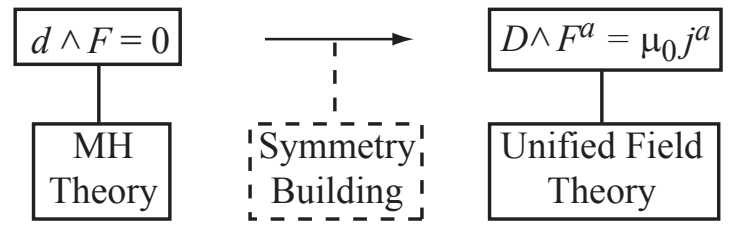

inhomogeneous field equation of the unified field theory is obtained by symmetry building as follows:

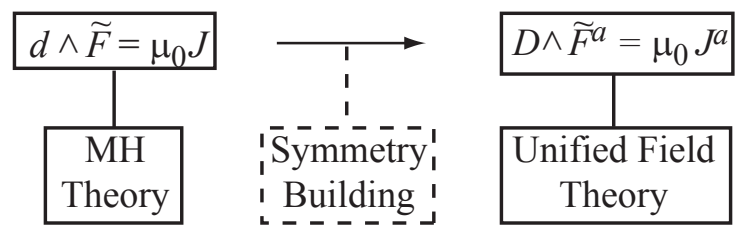

The MH field equations in this case are the Coulomb and the Ampère-Maxwell laws. So, to look for the effects of gravitation on electromagnetism, it is necessary to search experimentally for departures from these laws.

In tensor notation, the electromagnetic field in the Evans unified field theory is

$$
F_{\mu \nu}^{a}=A^{(0)} T_{\mu \nu}^{a}=A^{(0)} T_{\mu \nu}^{\kappa} q_{\kappa}^{a},
$$

where $T_{\mu \nu}^{\kappa}$ is the torsion tensor. Therefore, the inhomogeneous field equation of the unified field theory is

$$
D^{\mu} F_{\mu \nu}^{a}=\mu_{0} J_{\nu}^{a}
$$

where the charge current density is defined by

$$
J_{\nu}^{a}:=\frac{A^{(0)}}{\mu_{0}} q_{\kappa}^{a} D^{\mu}\left(\Gamma_{\mu \nu}^{\kappa}-\Gamma_{\nu \mu}^{\kappa}\right) .
$$

In differential-form notation, Eq. (42) reads

$$
D \wedge \widetilde{F}^{a}=\mu_{0} J^{a},
$$

and so the electromagnetic sector of the Evans unified field theory is described by

$$
D \wedge F^{a}=\mu_{0} j^{a},
$$




$$
D \wedge \widetilde{F}^{a}=\mu_{0} J^{a} .
$$

The unified field theory evolves from the original MH field theory by building symmetry as follows:

$$
\begin{aligned}
& d \wedge F=0, \\
& d \wedge \widetilde{F}=\mu_{0} J .
\end{aligned} \longrightarrow \quad \begin{aligned}
& D \wedge F^{a}=\mu_{0} j^{a}, \\
& D \wedge \widetilde{F}^{a}=\mu_{0} J .
\end{aligned}
$$

The symmetry of the unified field equation is that of the Einstein group with sixteen irreducible representations. This is a higher symmetry than that of $\mathrm{O}(3)$ electrodynamics, the intermediate theory in which the electromagnetic and gravitational fields are still decoupled, and $\mathrm{O}(3)$ electrodynamics has in turn a higher symmetry than U(1) electrodynamics, the MH symmetry. The experimental evidence for $\mathrm{O}(3)$ electrodynamics is summarized in Ref. [17].

In tensor notation, Eq. (23) reads

$$
\begin{gathered}
\partial_{\mu} T_{\nu \rho}^{a}+\omega_{\mu b}^{a} T_{\nu \rho}^{b}+\partial_{\nu} T_{\rho \mu}^{a}+\omega_{\nu b}^{a} T_{\rho \mu}^{b} \\
+\partial_{\rho} T_{\mu \nu}^{a}+\omega_{\rho b}^{a} T_{\mu \nu}^{b}=R_{\mu \nu \rho}^{a}+R_{\nu \rho \mu}^{a}+R_{\rho \mu \nu}^{a}
\end{gathered}
$$

and is a balance of identities of differential geometry [21]. On the righthand side is the Bianchi identity used in gravitational general relativity [27], and the left-hand side is the covariant exterior derivative of the torsion form. In tensor notation, the homogeneous field equation of the unified field theory is obtained directly from Eqs. (47) and (1) and reads

$$
\begin{gathered}
\partial_{\mu} F_{\nu \rho}^{a}+\omega_{\mu b}^{a} F_{\nu \rho}^{b}+\partial_{\nu} F_{\rho \mu}^{a}+\omega_{\nu b}^{a} F_{\rho \mu}^{b} \\
+\partial_{\rho} F_{\mu \nu}^{a}+\omega_{\rho b}^{a} F_{\mu \nu}^{b}=A^{(0)}\left(R_{\mu \nu \rho}^{a}+R_{\nu \rho \mu}^{a}+R_{\rho \mu \nu}^{a}\right)
\end{gathered}
$$

Equation (48) governs the unified gravitational and electromagnetic fields. When the two fields are unified they interact and mutually influence each other. This process is governed by

$$
R_{\mu \nu \rho}^{a}+R_{\nu \rho \mu}^{a}+R_{\rho \mu \nu}^{a} \neq 0 .
$$

When there is no influence of gravitation on electromagnetism both the left-hand and right-hand sides of Eq. (48) vanish identically

$$
\begin{gathered}
R_{\mu \nu \rho}^{a}+R_{\nu \rho \mu}^{a}+R_{\rho \mu \nu}^{a}=0 . \\
\partial_{\mu} F_{\nu \rho}^{a}+\omega_{\mu b}^{a} F_{\nu \rho}^{b}+\partial_{\nu} F_{\rho \mu}^{a}+\omega_{\nu b}^{a} F_{\rho \mu}^{b}+\partial_{\rho} F_{\mu \nu}^{a}+\omega_{\rho b}^{a} F_{\mu \nu}^{b}:=0 .
\end{gathered}
$$


Eq. (50) is the familiar Bianchi identity of the gravitational field uninfluenced by the electromagnetic field, and Eq. (51) is the homogeneous field equation of the electromagnetic field uninfluenced by the gravitational field.

The electromagnetic field is therefore the spinning of spacetime described by the torsion tensor and the gravitational field is the curving of spacetime described by the curvature tensor. Differential geometry is needed to build the symmetry of the unified field theory and in differential geometry spinning is described by the torsion form and curvature by the curvature form. The homogeneous field equation of the unified field is an identity, Eq. (48), of differential geometry, which is valid for a spacetime in which there is both spin and curvature. Such an identity is not available in Riemann geometry when the Christoffel connection is symmetric in its lower two indices. This is the type of spacetime used in the Einstein theory of gravitation, and in this spacetime Eq. (50) always holds. So such a spacetime cannot be used to unify gravitation and electromagnetism. The Maxwell-Heaviside field theory is philosophically different from the Einstein field theory in that the electromagnetic field is superimposed on a flat (Minkowski) spacetime in which the spin connection vanishes. In the Evans unified field theory this fundamental philosophical difference (which prevents field unification in the standard model and string theory) is bridged by recognizing that the electromagnetic field is the torsion form of differential geometry, so electromagnetism is the spinning of spacetime itself. This spinning is in general influenced by the curving of spacetime through Eqs. (47) or (48). We therefore obtain field unification by symmetry building. The intermediate stage in this process, $\mathrm{O}(3)$ electrodynamics, has been tested extensively against experimental data [17].

\section{EXPERIMENTAL TESTS OF THE EVANS THEORY}

It has been established and well accepted [1-20] that the Evans field theory infers the inverse Faraday effect through the Evans spin field

$$
B^{(3)}=-i g A^{(1)} \wedge A^{(2)},
$$

which is dual to torsion two-form of spacetime. The inverse Faraday effect therefore originates in the torsion or twisting motion of spacetime. In order to test this hypothesis accurately it is suggested that a high precision inverse Faraday effect calibrating experiment first be carried out as follows in a non-relativistic electron beam.

The inverse Faraday effect is the orbital angular momentum imparted to the electron beam by the Evans spin field. For a nonrelativistic electron beam, the energy exchanged during the process is [1-20]

$$
E n_{I F E}=\frac{e \hbar}{2 m} B^{(3)}=\frac{e^{2} A^{(0) 2}}{2 m}=\frac{p^{2}}{2 m} \text {. }
$$


Here $m$ is the mass of the electron,

$$
m=9.10953 \times 10^{-31} \mathrm{~kg},
$$

and $p=e A^{(0)}$ its linear momentum. Therefore, the energy imparted to the electron beam by the circularly polarized electromagnetic beam is the Newtonian kinetic energy $p^{2} / 2 m$. The energy in Eq. (53) may be written as [1-20]

$$
E n_{I F E}=\frac{e^{2} A^{(0) 2}}{2 m}=\left(\frac{e^{2} \mu_{0} c}{2 m}\right) \frac{I}{\omega^{2}},
$$

where $I$ is the beam power density $\left(W^{-2}\right)$ and $\omega$ is the beam angular frequency $\left(\mathrm{rads}^{-1}\right)$. The energy exchanged is characteristically dependent on the power density and the inverse square of the angular frequency.

The orbital angular momentum $J$ imparted to the electron beam can be calculated from the fundamental relation between energy and angular momentum,

$$
E n=\frac{1}{2} \omega J,
$$

and so

$$
J=\left(\frac{e^{2} \mu_{0} c}{2 m}\right) \frac{I}{\omega^{3}} .
$$

From Eq. (57) the magnetic flux density is tesla $\left(1.0 \mathrm{~T}=10^{4} \mathrm{G}\right) \mathrm{im}-$ parted to the non-relativistic electron beam may be calculated [1-20] to be

$$
B_{\text {sample }}^{(3)}=\mu_{0} M_{\text {sample }}^{(3)}=\frac{N}{V} \mu_{0} \frac{e}{2 m} J=\frac{N}{V}\left(\frac{\mu_{0}^{2} e^{3} c}{2 m^{2}}\right) \frac{I}{\omega^{3}} .
$$

Here $M^{(3)}$ is the magnetization produced in the inverse Faraday effect for $N$ electrons in a sample volume $V$; see Eq. (F5), p. 208, The Enigmatic Photon, Vol. 3 (Ref. 15). For a pulsed Nd YaG laser with $I=5.5 \times 10^{12} \mathrm{Wm}^{-2}, \omega=1.77 \times 10^{16} \mathrm{rads}^{-1}$ and $\mathrm{N} / \mathrm{V}=10^{26}$ electrons $m^{-3}$ (Avogadro's number), we find

$$
B_{\text {sample }}^{(3)} \sim 10^{-9} \mathrm{~T}=10^{-5} \mathrm{G},
$$

an order of magnitude observed experimentally in liquids, solids and gases, for example. The inverse Faraday effect has been observed many times [1-20] in a variety of materials, and solidly indicates the existence of the Evans spin field $\mathbf{B}^{(3)}$. However a high precision, baseline experiment in a non-relativistic electron beam has yet to be carried out. 
Having carried out this baseline experiment, it is then necessary to develop radiatively induced fermion resonance (RFR), which originates in the spin angular momentum imparted to the electron beam by the Evans spin field [1-20]:

$$
\sigma_{0} E n_{R F R}=\frac{e \hbar}{2 m} B^{(3)} \sigma_{Z}=\frac{e^{2} A^{(0) 2}}{2 m} \sigma_{Z}=\frac{p^{2} \sigma_{Z}}{2 m} .
$$

Here $\sigma_{Z}$ is the third Pauli matrix and $\sigma_{0}$ the zero-order Pauli matrix. It is seen from Eq. (60) that RFR originates in the Newtonian kinetic energy multiplied by $\sigma_{Z}$. Electron spin resonance between the states of $\sigma_{Z}$ gives rise to RFR as follows:

$$
E n=2 \pi \hbar f_{\mathrm{res}}=\frac{e^{2} A^{(0)}}{2 m}[1-(-1)]=\left(\frac{e^{2} \mu_{0} c}{m}\right) \frac{I}{\omega^{2}},
$$

so the resonance frequency in hertz is

$$
f_{\mathrm{res}}=\left(\frac{e^{2} \mu_{0} c}{2 \pi \hbar m}\right) \frac{I}{\omega^{2}}=1.007 \times 10^{28} \frac{I}{\omega^{2}} .
$$

It displays the characteristic $I / \omega^{2}$ dependence of the IFE (Eq. (55)). This property of RFR means that it can produce fermion resonance at high resolution without magnets, for example electron spin resonance, nuclear magnetic resonance and magnetic resonance imaging without permanent magnets [1-20]. The magnet is replaced by a circularly polarized radio frequency beam.

Thirdly, it is necessary to carry out an accurate test of EMAB by replacing the magnetized iron whisker of the original Chambers experiment by a circularly polarized radio frequency beam. The EMAB is caused by the $\mathbf{B}^{(3)}$ of the radio frequency field and detected as a shift in the fringe pattern of two interfering electron beams:

$$
\Delta x=\frac{L}{d} \frac{\lambda}{2 \pi} \Delta \delta
$$

in a Young interferometer. Here $L$ is the distance between the plates of the interferometer, $d$, the distance between the openings in the first plate, and $\lambda$ the electron wavelength. In the original Chambers experiment used to detect the Aharonov-Bohm effect:

$$
\Delta \delta=\frac{e}{\hbar} B A r=\frac{e}{\hbar} \Phi,
$$

where $B$ is the magnetic flux density produced by the iron whisker and $A r$ the area enclosed by the electron beams. This shift was first observed by Chambers in 1959 following the original theory by Aharanov 
and Bohm. This has recently been understood in terms of the Evans phase law [1-20]:

$$
\Delta \phi=\exp \left(i \frac{e}{\hbar} \int \mathbf{B}^{(3)} \cdot \mathbf{k} d A r\right)
$$

which follows from the fact that any magnetic field must be defined by

$$
B=D \wedge A=d \wedge A+g A \wedge A,
$$

where $g=e / \hbar$. In the complex circular basis, Eq. (66) becomes

$$
B^{(3)}=-i g A^{(1)} \wedge A^{(2)} .
$$

The conventional magnetic field is described by Eq. (66), when $B$ is the magnetic flux density of the iron whisker [1-20]. So the Evans field theory gives a consistent description both of the conventional and electromagnetic Aharonov-Bohm effects in terms of the covariant derivative (66).

In vector notation,

$$
\mathbf{B}^{(3)}=-i g \mathbf{A}^{(1)} \times \mathbf{A}^{(2)},
$$

and the magnitude of the field is

$$
B^{(3)}=\left|\mathbf{B}^{(3)}\right|=\frac{e}{\hbar} A^{(0) 2},
$$

where

$$
A^{(0) 2}=\mu_{0} c I / \omega^{2},
$$

showing again the characteristic $I / \omega^{2}$ dependence. In SI units:

$$
\begin{aligned}
\mu_{0} & =4 \pi \times 10^{-7} J^{2} C^{-2} m^{-1}, c=2.997925 \times 10^{8} \mathrm{~ms}^{-1}, \\
e & =1.60219 \times 10^{-19} C, \hbar=1.05459 \times 10^{-34} \mathrm{Js},
\end{aligned}
$$

and

$$
B^{(3)}=\left(\frac{e \mu_{0} c}{\hbar}\right) \frac{I}{\omega^{2}} .
$$

From Eqs. (64) and (72):

$$
\frac{\Delta \delta}{A r}=\left(\frac{e^{2} \mu_{0} c}{\hbar^{2}}\right) \frac{I}{\omega^{2}}=8.6954 \times 10^{34} \frac{I}{\omega^{2}},
$$

which is the phase shift per unit area enclosed by the electron beams due to a circularly polarized radio frequency field replacing the iron 
whisker in the Chambers experiment. This is the electromagnetic Aharanov-Bohm (EMAB) effect. effect is

The equivalent of Eq. (73) for the magnetic Aharonov-Bohm

$$
\frac{\Delta \delta}{A r}=\frac{e}{\hbar} B
$$

for a magnetic field $1 \mathrm{G}\left(=10^{-4} \mathrm{~T}\right)$, and the phase shift per unit area from Eq. (74) is $1.519 \times 10^{11} \mathrm{~m}^{-2}$.

For an electromagnetic field of $1.0 \mathrm{G} \mathrm{Hz}$ angular frequency and $10^{-4} \mathrm{Wm}^{-2}$ power density (1 watt per $\mathrm{cm}^{2}$ ) the phase shift per unit area from Eq. (73) is $8.695 \times 10^{12} \mathrm{~m}^{-2}$. Under such conditions, the EMAB effect should be observable using a modification of the Chambers experiment. However, if a Nd YaG laser is used at the same power density but at angular frequency $1.77 \times 10^{16} \mathrm{rads}^{-1}$, the phase shift per unit area becomes $0.013 \mathrm{~m}^{-2}$ and unobservable. An EMAB effect might however be observable with a $\mathrm{Nd}$ YaG laser if the latter is pulsed to very high power densities.

The observation of the IFE in this way would be important for fundamental physics, the observation of the RFR would open up a new industry, and the observation of the EMAB effect would lead to several new radar technologies.

Acknowledgments. Craddock Inc., Applied Science Associates, and the Ted Annis Foundation are thanked for funding.

\section{REFERENCES}

1. M. W. Evans, "Derivation of $\mathrm{O}(3)$ electrodynamics from generally covariant unified field theory," Found. Phys. Lett. submitted; preprint on www.aias.us.

2. M. W. Evans, Found. Phys. Lett. 16, 367 (2003).

3. M. W. Evans, Found. Phys. Lett. 16, 507 (2003).

4. M. W. Evans, Found. Phys. Lett. 17, 25 (2004).

5. M. W. Evans, Found. Phys. Lett. 17, 149 (2004).

6. M. W. Evans, Found. Phys. Lett. 17, 267 (2004).

7. M. W. Evans, Found. Phys. Lett. 17, 301 (2004).

8. M. W. Evans, Found. Phys. Lett. 17, 393 (2004).

9. M. W. Evans, "The Evans lemma of differential geometry, Found. Phys. Lett. 17, in press (2004); preprint on www.aias.us.

10. M. W. Evans, "Derivation of the Evans wave equation from the Lagrangian and action. Origin of the Planck constant in general relativity," Found. Phys. Lett. 17, 535 (2004); preprint on www.aias.us. 
11. M. W. Evans and the AIAS Author Group, "Development of the Evans wave equations in the weak-field limit," Found. Phys. Lett. 17, 497 (2004); preprint on www.aias.us.

12. M. W. Evans, "New concepts from the Evans unified field theory. Part One: The evolution of curvature, oscillatory universe without singularity, causal quantum mechanics, and general force and field equations," Found. Phys. Lett., submitted (2004); preprint on www.aias.us.

13. M. W. Evans, "New concepts from the Evans unified field theory. Part Two: Derivation of the Heisenberg equation and replacement of the Heisenberg uncertainty principle," Found. Phys. Lett., submitted (2004); preprint on www.aias.us.

14. M. W. Evans, "Derivation of the Lorentz-boost from the Evans wave equation," Found. Phys. Lett., in press (2004).

15. M. W. Evans et al., The Enigmatic Photon, Vols. 1-5 (Kluwer Academic, Dordrecht, 1994 to 2002, hardback and softback).

16. M. W. Evans, Generally Covariant Unified Field Theory: The Geometrization of Physics (Kluwer Academic, 2004, in press). M. W. Evans and L. Felker, The Evans Equations of Unified Field Theory (World Scientific, Singapore, 2004, in preparation).

17. M. W. Evans, ed., Modern Non-Linear Optics, a special topics issue of I. Prigogine and S. A. Rice, series eds., Advances in Chemical Physics (Wiley-Interscience, New York, 2001, 2nd and e-book edns.), Vols. 119(1) to 119(3); ibid., 1st edn., Vol. 85.

18. M. W. Evans and L. B. Crowell, Classical and Quantum Electrodynamics and the $\mathbf{B}^{(3)}$ Field (World Scientific, Singapore, 2001).

19. M. W. Evans and A. A. Hasanein, The Photomagneton in Quantum Field Theory (World Scientific, Singapore, 1994).

20. M. W. Evans, Physica B 182, 227, 237 (1992).

21. S. P. Carroll, Lecture Notes in General Relativity (University of California, Santa Barbara, graduate course), arXiv:grgq/9712019 vl 3 Dec 1997).

22. L. H. Ryder, Quantum Field Theory, 2nd edn. (University Press, Cambridge, 1996).

23. A. Einstein, The Meaning of Relativity (Princeton University Press, 1921). 